\title{
IMPLEMENTAC̣̃̃O DO PROTOCOLO TRS-398 PARA FEIXES DE ELÉTRONS NO HOSPITAL ISRAELITA ALBERT EINSTEIN*
}

\author{
Laura Natal Rodrigues ${ }^{1}$, José Carlos da Cruz ${ }^{2}$, Paulo José Cecílio ${ }^{3}$, Lourenço Caprioglio ${ }^{3}$
}

Resumo OBJETIVO: A fim de simplificar a utilização das tabelas correspondentes aos fatores de qualidade no TRS398 da International Atomic Energy Agency na calibração cruzada da câmara de placas paralelas por comparação com uma câmara cilíndrica calibrada em um feixe de elétrons de qualidade $\boldsymbol{Q}_{\text {cross }}$, é introduzida uma energia intermediária e arbitrária $Q_{i n t}$ na qual a câmara de placas paralelas deverá ser calibrada. 0 objetivo deste trabalho consiste em avaliar a escolha desta energia intermediária. MATERIAIS E MÉTODOS: Uma câmara de placas paralelas da Scanditronix, modelo NACP-02, e uma câmara PTW Markus foram calibradas em dois aceleradores lineares da Varian, Clinac 2100C e Clinac 23EX, nas energias de 16 e 20 MeV, respectivamente. Como câmara de referência foi utilizada uma câmara da Nuclear Enterprises, modelo 2571, previamente calibrada em termos de $D_{w}$ no Instituto de Pesquisas Energéticas e Nucleares, para um feixe de ${ }^{60}$ Co. RESULTADOS: Os fatores de calibração $N_{D, w}$ assim obtidos apresentam uma variação de $0,07 \%, 0$ que pode ser considerado desprezível. Os valores de dose absorvida na água determinados no Clinac $2100 \mathrm{C}$ para uma $Q_{\text {int }}$ de $16 \mathrm{MeV}$ apresentam uma variação de 0,04\% para a menor energia (4 MeV) e de 2,6\% para a maior energia (16 MeV), em relação ao protocolo TRS-381. Já para uma $Q_{\text {int }}$ de $20 \mathrm{MeV}$, as variações observadas são de 1,3\% e 3,5\%. Por outro lado, as doses determinadas no Clinac 23EX para as $Q_{\text {int }}$ de 16 MeV e de $20 \mathrm{MeV}$ são de 1,9\% e 2,0\% e de 2,8\% e 3,0\%, respectivamente. CONCLUSÃO: A escolha de $Q_{i n t}$ não é tão crítica assim, principalmente quando não de dispõem de feixes de elétrons que tenham na prática um valor de $R_{50}$ exatamente igual a $7,5 \mathrm{~g} \cdot \mathrm{cm}^{-2}$.

Unitermos: Calibração de câmaras de placas paralelas; Dosimetria de feixe de elétrons; Protocolos de dosimetria.

Abstract Implementation of TRS-398 protocol for electron beams at "Hospital Israelita Albert Einstein", São Paulo, Brazil.

OBJECTIVE: In order to simplify the use of the corresponding tables for the quality factors of International Atomic Energy Agency TRS-398, the cross-calibration of parallel chambers is performed by comparison with a cylindrical chamber calibrated at a quality electron beam $Q_{\text {cross }}$; an intermediate and random energy $Q_{i n t}$ is introduced in which the parallel chamber will be calibrated. The objective of this study is to evaluate the selection of this intermediate energy. MATERIALS AND METHODS: A parallel chamber from Scanditronix, model NACP-02 and a PTW Markus chamber were calibrated in two linear accelerators from Varian, Clinac $2100 \mathrm{C}$ and Clinac 23EX, in the energies of 16 and $20 \mathrm{MeV}$ respectively. A Nuclear Enterprises chamber, model 2571 previously calibrated in terms of $D_{w}$ at "Instituto de Pesquisas Energéticas e Nucleares", São Paulo, Brazil, for a ${ }^{60} \mathrm{Co}$ beam was used as reference. RESULTS: The calibration factors $N_{D, w}$ thus obtained show a variation of $0.07 \%$ that can be considered negligible. The absorbed dose values in water determined at Clinac $2100 \mathrm{C}$ for a $Q_{\text {int }}$ of $16 \mathrm{MeV}$ present a variation of $0.04 \%$ for the smallest energy (4 MeV) and of $\mathbf{2 . 6 \%}$ for the largest energy $(16 \mathrm{MeV})$, in relation to the TRS-381 protocol. For a $Q_{\text {int }}$ of $20 \mathrm{MeV}$, the observed variations are of $1.3 \%$ and $3.5 \%$, respectively. On the other hand, the doses obtained in Clinac23EX for $Q_{\text {int }}$ of $16 \mathrm{MeV}$ and of $20 \mathrm{MeV}$ are of $1.9 \%$ and $2.0 \%$ and of $2.8 \%$ and $3.0 \%$ respectively. CONCLUSION: The choice of $Q_{\text {int }}$ is not so critical, particularly when no electron beams which have a $R_{50}$ value exactly equal to $7.5 \mathrm{~g} . \mathrm{cm}^{-2}$ are available.

Keywords: Calibration of parallel chambers; Electron beams dosimetry; Dosimetry protocols.

* Trabalho realizado no Hospital Israelita Albert Einstein, São Paulo, SP.

1. Instituto de Pesquisas Energéticas e Nucleares (Ipen) Doutora em Biofísica pela Universidade Federal do Rio de Janeiro (UFRJ).

2. Hospital Israelita Albert Einstein, Doutor em Medicina pela Universidade Federal de São Paulo (Unifesp).

3. Físicos Médicos do Departamento de Radioterapia do Hospital Israelita Albert Einstein.

Endereço para correspondência: Laura Natal Rodrigues. Rua Desembargador do Vale, 900, ap. 114, Perdizes. São Paulo, SP, 0510-040. E-mail: Inatal@ipen.br

Recebido para publicação em 6/5/2005. Aceito, após revisão, em 7/6/2005.

\section{INTRODUÇÃO}

O objetivo principal da radioterapia é liberar uma distribuição de dose curativa para os tecidos-alvo, a fim de erradicar todas as suas células clonogênicas, mantendo, ao mesmo tempo, as reações agudas e tardias nos tecidos sadios e normais em um nível mínimo. Esta é uma tarefa árdua, já que é impossível liberar dose somente ao volume-alvo sem afetar os tecidos sadios, sobretudo no caso de tumores profundos, que são tratados por feixes externos. Dessa forma, o objetivo primário da radioterapia é liberar uma dose suficientemente alta para as células tumorais, mantendo, ao mesmo tempo, a dose nos tecidos normais circunvizinhos o mais baixo possível.

A publicação da International Comission on Radiation Units (ICRU) ${ }^{(\mathbf{1})}$ concluiu 
que existe a evidência para certos tipos de tumores que a precisão requerida na determinação da dose absorvida no volume-alvo deve estar dentro de limites de incerteza de $\pm 5 \%$ ou até menos caso seja pretendida a erradicação do tumor. $\mathrm{O}$ requisito de tal precisão pode ser considerado como uma tolerância para o desvio entre a dose prescrita e a dose liberada no alvo. Conseqüentemente, existe grande necessidade de aperfeiçoamento na dosimetria.

Desde 1978, o Comitê Alemão de Padrões (Normenausschuss Radiologie im DIN - Deutsches Institut für Normung) propôs formalmente a calibração de instrumentos de campo em termos de "dose absorvida na água" para toda faixa de energia de feixes de fótons utilizados em radioterapia $^{(2)}$. Entretanto, esta aproximação não foi adotada na época, uma vez que não existia a disponibilidade de nenhum padrão de dose absorvida na água.

A dose absorvida na água é a grandeza de interesse principal em radioterapia, já que relaciona o mais próximo possível os efeitos biológicos produzidos pela radiação ionizante. A vantagem principal de se ter tal grandeza como padrão básico é que a mesma grandeza é empregada ao longo de toda a cadeia metrológica, desde os padrões primários até os instrumentos de campo. Além do mais, a grandeza (dose absorvida), em termos da qual os padrões primários determinam as suas leituras, estará próxima do efeito físico no qual o padrão se baseia, o que minimizará a aplicação de vários fatores de correção, assim como as incertezas envolvidas neste procedimento. É importante ressaltar que este conceito de calibração em termos de dose absorvida na água se aplica a todos os tipos de radiação e para todas as energias.

Seguindo este conceito, vários laboratórios de calibração (Bureau International des Poids et Mesures - BIPM, National Physical Laboratory - NPL, National Research Council Canada - NRC e Physikalisch-Technische Bundesanstalt - PTB) desenvolveram ou estão desenvolvendo calorímetros como padrão primário para dose absorvida na água em raios- $\gamma$ de ${ }^{60} \mathrm{Co}$, bem como em aceleradores lineares. A primeira etapa neste procedimento é fornecer o fator de calibração em termos de dose absorvida na água para os usuários no feixe de referência, geralmente raios- $\gamma$ de ${ }^{60} \mathrm{Co}$. A segunda etapa consiste na determinação dos fatores de correção da câmara que dependem da qualidade do feixe para várias energias.

Simultaneamente, surgiu uma proposta de um novo formalismo para a determinação da dose absorvida na água ${ }^{(\mathbf{3})}$. Neste formalismo, as etapas para a determinação da dose são bastante simplificadas e, conseqüentemente, as incertezas envolvidas são menores. Desde a publicação do DIN $6800^{(4)}$, alguns Laboratórios já estão utilizando este formalismo com o objetivo de determinar a dose absorvida na água. Além disso, foram realizadas intercomparações anuais visando à aplicação prática deste novo formalismo, assim como estabelecer a estabilidade a longo prazo de seus padrões de dose absorvida.

Desde a publicação do protocolo nórdico de dosimetria ${ }^{(\mathbf{5})}$, a maioria dos protocolos de dosimetria tem baseado suas recomendações para a determinação da dose absorvida na água em feixes de elétrons pelo uso de câmaras de ionização de placas paralelas. A aproximação mais comum $^{(6)}$ requer um fator de calibração em termos de dose absorvida na água $N_{D, w}$, obtido por calibração cruzada. Esta calibração é realizada em um feixe de elétrons de alta energia com relação a uma câmara cilíndrica de referência com o fator de calibração $N_{D, w}$ conhecido e fornecido por um laboratório de dosimetria padrão.

De acordo com as recomendações do protocolo atual da International Atomic Energy Agency (IAEA) ${ }^{(7)}$, uma das alternativas para a calibração de câmaras de placas paralelas diretamente em feixes de elétrons de alta energia consiste na calibração em uma série de qualidades de feixes de elétrons. Todavia, devido à disponibilidade limitada de tais calibrações por poucos laboratórios primários, a aproximação a ser empregada consiste na calibração cruzada da câmara de placas paralelas por comparação com uma câmara cilíndrica calibrada em um feixe de elétrons de qualidade $Q_{\text {cross. }}$. A fim de simplificar a utilização das tabelas correspondentes aos fatores de qualidade, é introduzida uma energia intermediária e arbitrária $Q_{i n t}$, correspondente a um $R_{50}$ de 7,5 g.cm ${ }^{-2}$, na qual a câmara de placas paralelas deverá ser calibrada.
O objetivo deste trabalho consiste em avaliar a escolha desta energia intermediária, bem como a influência deste fator de calibração assim obtido na determinação da dose absorvida nas demais energias disponíveis no hospital para fins de calibração dos feixes terapêuticos.

\section{MATERIAIS E MÉTODOS}

De acordo com este novo formalismo da $\operatorname{IAEA}^{(7)}$, a determinação da dose absorvida na água é dada simplesmente pelo produto da leitura da câmara de ionização, pelo fator de calibração em termos da dose absorvida na água e pelo produto dos fatores de correção. Estes fatores de correção levam em consideração todos os efeitos devidos às grandezas de influência que diferem do valor de referência. Isto pode ser obtido experimentalmente pela comparação da câmara de ionização com o padrão primário, sob condições de referência.

As grandezas de influência são aquelas grandezas que não são objeto de medida, mas influenciam o valor medido, tais como temperatura, umidade, pressão, tensão de polarização, taxa de dose e qualidade da radiação. A qualidade da radiação, no documento DIN 6800 ${ }^{(4)}$, é considerada explicitamente como grandeza de influência. A radiação gama de ${ }^{60} \mathrm{Co}$ é considerada como qualidade de referência, e medidas em outras qualidades requerem um fator de correção. Este tratamento formal resulta em apenas um fator de calibração para a câmara de ionização, e os fatores de correção estão relacionados apenas com uma grandeza de influência.

Assim, pode-se constatar que este procedimento elimina as incertezas associadas com a conversão de um fator de calibração em termos de kerma no ar para um fator de calibração em termos de dose absorvida na água, uma vez que este procedimento utiliza apenas uma relação fundamental, reduzindo, conseqüentemente, a probabilidade de erros pelo usuário no hospital.

A câmara cilíndrica de referência empregada foi uma câmara tipo Farmer da Nuclear Enterprises, modelo 2571, número de série 2194. Esta câmara foi previamente calibrada em um feixe de ${ }^{60} \mathrm{Co}$ no Laboratório de Calibração de Instrumentos do Instituto de Pesquisas Energéticas e 
Nucleares (Ipen). Esta câmara de ionização com volume sensível de $0,6 \mathrm{~cm}^{3}$ consiste de uma parede fina de grafite de alta pureza em forma de dedal (espessura de 0,0065 g.cm ${ }^{-2}$ ) e um eletrodo de alumínio puro (sólido), sustentada por uma haste fina de alumínio. Possui, também, uma capa de equilíbrio eletrônico de Delrin com 0,551 g. $\mathrm{cm}^{-2}$ de espessura ${ }^{(8)}$.

Um dos primeiros protocolos de dosimetria a abordar o uso e a calibração de câmaras de placas paralelas foi o da Nordic Association of Clinical Physics (NACP) ${ }^{(\mathbf{5})}$. Esse documento estabelecia o procedimento de medida para a determinação da dose absorvida na água $\left(D_{w}\right)$ para energias médias em feixes de elétrons na superfície do objeto simulador para energias $E_{o}$ $<15 \mathrm{MeV}$.

Os principais requisitos de uma câmara de placas paralelas para dosimetria de feixes de elétrons de alta energia são os seguintes: a cavidade deve ser pequena na direção do feixe; o anel de guarda deve ser largo o suficiente para que a perturbação decorrente do efeito de espalhamento interno seja insignificante, ou seja, elétrons espalhados provenientes da parede lateral da cavidade não contribuem significativamente no sinal, até mesmo para feixes de elétrons de baixa energia.

Com relação às câmaras de placas paralelas, foram utilizados dois tipos de câmaras. A primeira câmara é uma câmara Markus da PTW, modelo 23343, número de série 1369 . A outra câmara é uma câmara NACP da Scanditronix, modelo 02, número de série 34-03.

A câmara Markus ${ }^{(9)}$ tem uma parede de 0,9 mm de espessura contituída de uma fotha de polietileno grafitado, um eletrodo coletor de poliestireno grafitado. O corpo externo da câmara é de polimetilmetacrilato (PMMA) com uma capa de PMMA também, que torna a câmara imersível na água. A espessura desta capa está incluída na espessura total da parede frontal da câmara. $\mathrm{O}$ espaçamento entre os eletrodos é de $2 \mathrm{~mm}$ e o comprimento do anel de guarda é de $0,2 \mathrm{~mm}$.

A câmara NACP tem uma parede frontal de $0,5 \mathrm{~mm}$ de espessura feita de grafite ${ }^{(5)}$. A câmara NACP modelo 02 usada neste estudo tem uma folha adicional de "mylar" (0,1 mm) na parede frontal, o que a torna à prova d'água. O eletrodo coletor consiste de um revestimento bem fino de grafite sobre um isolante fino de poliestireno. $\mathrm{O}$ isolante é colocado em um bloco de grafite conectado ao sistema de guarda. O eletrodo coletor é rodeado por um anel de guarda. O corpo externo da câmara é feito de rexolite. $\mathrm{O}$ pequeno espaçamento de ar (2 $\mathrm{mm})$ e a presença de um anel de guarda minimizam os efeitos de perturbação da câmara ${ }^{(\mathbf{1 0})}$. O comprimento do anel de guarda desta câmara é de $3 \mathrm{~mm}$, comparativamente com o comprimento de 0,2 mm da câmara Markus.

Estas duas câmaras de placas paralelas foram então calibradas no Hospital Israelita Albert Einstein em dois aceleradores lineares da Varian, Clinac 2100C e Clinac 23EX, nas energias de 16 e $20 \mathrm{MeV}$, respectivamente. As medidas foram feitas na água com um sistema de posicionamento automático da Scanditronix, modelo RFA7C, número de série 1100-002, com um objeto simulador feito de PMMA, uma unidade auxiliar tridimensional e eletrômetros duplos para câmaras de ionização sob controle computacional, eliminando todos os ajustes manuais, além de indicar em tela o controle de "menu" dos programas, bem como um monitor gráfico colorido de alta resolução para apresentação dos dados medidos. A sua unidade de controle tem resolução de $0,1 \mathrm{~mm}$ em termos de posicionamento e de $0,1 \%$ no que diz respeito às leituras normalizadas (Scanditronix, System Operation Manual, Uppsala, Sweden).

Aplicando a metodologia da calibração cruzada, o fator de calibração da câmara de placas paralelas é obtido pela igualdade entre os valores de dose absorvida na água, medidas em um mesmo ponto de referência ${ }^{(9)}$. Para tal, é preciso assegurar o posicionamento correto de ambas as câmaras, tanto a câmara cilíndrica de referência (fator $N_{D, w}$ conhecido) quanto a câmara de placas paralelas a ser calibrada. Este método é preferível e recomendado pelo protocolo de dosimetria atual ${ }^{(7)}$, uma vez que fornece uma dosimetria consistente, tanto para alta quanto para baixa energia. Neste caso, o método de câmara de placas paralelas é forçado a fornecer a mesma calibração da dose monitor no acelerador, assim como o método da câmara cilíndrica, pelo menos para uma energia.

\section{RESULTADOS E DISCUSSÃO}

Devido à falta de disponibilidade de uma calibração direta em feixes de elétrons de alta energia, até mesmo pela ausência de padrões primários para tais feixes, a calibração cruzada de uma câmara de placas paralelas por comparação com uma câmara cilíndrica calibrada em uma qualidade $Q_{\text {cross }}$ é a melhor alternativa ${ }^{(7)}$. De acordo com o novo formalismo da IAEA, os fatores de qualidade $k_{Q, Q_{\text {cross }}}$ possibilitarão o uso subseqüente desta câmara assim calibrada em feixes de elétrons de qualidade $Q$. Entretanto, tal utilização não é trivial, uma vez que a qualidade $Q_{\text {cross }}$ da calibração cruzada não é única, de tal forma que para um dado tipo de câmara será necessário empregar uma tabela bidimensional de fatores $k_{Q, Q_{\text {cross }}}$.

Esta situação pode ser contornável pela introdução de uma qualidade arbitrária $Q_{i n t}$ e que é uma qualidade intermediária entre a qualidade da calibração cruzada $Q_{\text {cross }}$ e a qualidade do usuário $Q$. Neste caso, o fator de qualidade $k_{Q, Q_{\text {cross }}}$ requerido é estimado a partir da seguinte relação:

$$
k_{Q, Q_{\text {cross }}}=\frac{k_{Q, Q_{i n t}}}{k_{Q_{\text {cross }}, Q_{i n t}}}
$$

O termo no denominador corrige o fator de calibração atual da câmara $N_{D, w, Q_{c r o s s}}$ para o fator de calibração que se aplica na qualidade intermediária $Q_{i n t}$. Já o numerador corrige este último fator de calibração que se aplica na qualidade $Q$ do usuário, de forma a tornar aplicável o formalismo estabelecido pelo protocolo da IAEA. O valor escolhido para $Q_{i n t}$ é escolhido arbitrariamente neste protocolo, como sendo igual a $R_{50}>7$ g.cm ${ }^{-2}$.

Para a calibração cruzada, recomendase a utilização do feixe de maior energia disponível no acelerador linear, isto é, $R_{50}$ $>7 \mathrm{~g} \cdot \mathrm{cm}^{-2}\left(E_{o}>16 \mathrm{MeV}\right)$. A câmara a ser calibrada é comparada alternadamente posicionando cada câmara na profundidade $z_{\text {ref }}$ na água, de acordo com o descrito anteriormente.

O ponto de referência de uma câmara de placas paralelas é considerado como sendo a superfície interna da parede frontal da câmara, enquanto, para a câmara cilíndrica, o centro geométrico precisa ser 
posicionado a $0,5 r_{c i l}$ mais profundo do que o ponto de interesse no objeto simulador, em que $r_{c i l}$ é o raio interno da cavidade da câmara. Todas as câmaras de ionização estão tabeladas no protocolo da IAEA.

Dessa forma, o fator de calibração para câmara de placas paralelas será dado pela seguinte relação:

$$
N_{D, w, Q_{\text {cross }}}^{x}=\frac{M_{Q_{\text {cross }}}^{r e f}}{M_{Q_{\text {cross }}}^{x}} N_{D, w, Q_{o}}^{r e f} k_{Q_{\text {cross }}, Q_{o}}^{r e f}
$$

em que: $N_{D, w, Q_{o}}$ é o fator de calibração da câmara de referência na qualidade $Q_{o}$ (geralmente, o ${ }^{60} \mathrm{Co}$ ); e $k_{Q_{\text {cross }}, Q_{o}}$ é o fator de correção para a qualidade do feixe para a câmara de referência.

Normalmente, a qualidade de referência será o ${ }^{60}$ Co e o valor de $k_{Q_{\text {cross }} Q_{o}}$ será obtido a partir da Tabela 18 do TRS-398 ${ }^{(7)}$. No caso em que $Q_{o}$ for o feixe de elétrons de alta energia, o valor de $k_{Q_{\text {cross }}, Q_{o}}$ precisa ser obtido de acordo com a seguinte expressão:

$$
k_{Q_{\text {cross }}, Q_{o}}^{r e f}=\frac{k_{Q_{c r o s s}, Q_{i n t}}^{r e f}}{k_{Q_{o}, Q_{i n t}}^{r e f}}
$$

em que tanto o numerador quanto o denominador serão obtidos a partir da Tabela 19 do TRS-398 ${ }^{(7)}$.

As duas câmaras de placas paralelas, câmara Markus e câmara NACP, empregadas neste estudo foram assim calibradas por comparação com uma câmara cilíndrica de referência. Esta câmara cilíndrica, tipo Farmer, câmara NE2571, número de série 2194, foi calibrada em termos de dose absorvida na água pelo Laboratório de Calibração de Instrumentos do Ipen em 2003. O fator de calibração emitido pelo certificado de calibração é de 4,643 Gy.nC ${ }^{-1}$, com uma incerteza de $0,7 \%$ (fator de cobertura $\mathrm{k}=2$ ).

A calibração cruzada foi então realizada no acelerador linear da Varian, Clinac 2100C, para um feixe de elétrons com energia de $16 \mathrm{MeV}$ e na profundidade de referência, de acordo com as recomendações do protocolo da IAEA $^{(7)}$. Os fatores de calibração $N_{D, w}$ assim obtidos são de 0,4887 e de 0,1417 Gy.nC ${ }^{-1}$ para as câmaras Markus e NACP, respectivamente. A fim de investigar uma possível dependência do fator $N_{D, w}$ com o acelerador linear e a energia, a calibração da câmara NACP foi também efetuada em um acelerador linear da Varian, Clinac 23EX, com energia de 20 $\mathrm{MeV}$. O fator de calibração encontrado é de 0,1416 Gy.nC ${ }^{-1}$.

Os fatores de calibração $N_{D, w}$ assim obtidos nos dois aceleradores apresentam uma variação de $0,07 \%$, o que pode ser considerado desprezível. Em uma segunda etapa, as duas câmaras de placas paralelas, Markus e NACP, foram empregadas na determinação da dose absorvida na água nas demais energias disponíveis no hospital para fins de calibração do feixe terapêutico. Os resultados obtidos nestes dois aceleradores e para as duas qualidades intermediárias analisadas são apresentados nas Tabelas 1 e 2 .

Observa-se que para a câmara Markus são encontradas variações na faixa de $0,07 \%$ a 4,4\% no Clinac $2100 \mathrm{C}$, e de $0,06 \%$ a 6,2\% no Clinac 23EX, para uma energia de calibração de $16 \mathrm{MeV}$. Já desde a publicação do protocolo da IAEA para feixes de elétrons de alta energia ${ }^{(9)}$ se apontava uma variação anormal do fator de perturbação da câmara Markus, comparativamente com as demais câmaras de placas pa- ralelas e que é devida a detalhes construcionais da câmara. A fim de contornar tal deficiência, o fabricante lançou, recentemente, uma nova câmara Markus, denominada comercialmente de Advanced Markus. Neste sentido, a IAEA já incorporou, em março de 2005, os fatores de correção para as qualidades dos feixes correspondentes a esta nova câmara Markus ${ }^{(11)}$.

Ao fazer a transição do protocolo anterior da IAEA $^{(8)}$ para o protocolo atual ${ }^{(7)}$, espera-se uma variação mínima de 2,5\% para a câmara Markus ${ }^{(\mathbf{1 2})}$. No protocolo anterior, assumia-se um valor unitário para o fator de perturbação, assim como para as demais câmaras. Já no protocolo específico para feixes de elétrons de alta energia ${ }^{(9)}$, o fator de perturbação da câmara Markus chega a 3\%, comparativamente com a câmara NACP, para uma energia média na profundidade $z$ de $3 \mathrm{MeV}$.

É importante ressaltar que os valores de $k_{16 \mathrm{MeV}, Q_{i n t}}^{x}$ e $k_{20 \mathrm{MeV}, Q_{i n t}}^{x}$ diferem de $0,9 \%$ para a câmara NACP e esta diferença será aplicada para todas as energias. Para validação final destes resultados, foi determinado o fator rendimento em termos de dose na água para a câmara cilíndrica de referên-

Tabela 1 Características dos feixes de elétrons do acelerador Clinac $2100 \mathrm{C}$ e calibração dos feixes de energias de referência de 16 e $20 \mathrm{MeV}$, respectivamente, usando as câmaras de placas paralelas Markus

\begin{tabular}{|c|c|c|c|c|c|c|c|c|}
\hline $\begin{array}{l}\text { Energia } \\
(\mathrm{MeV})\end{array}$ & $\begin{array}{c}R_{50} \\
\text { (g. } \mathrm{cm}^{-2} \text { ) }\end{array}$ & $\begin{array}{c}z_{r e f} \\
\text { (g. } \mathrm{cm}^{-2} \text { ) }\end{array}$ & $\begin{array}{c}\text { PDP } \\
\text { em } z_{\text {ref }}\end{array}$ & $\begin{array}{c}k_{Q, Q_{\text {int }}} \\
\text { Markus }\end{array}$ & $\begin{array}{l}k_{\mathrm{Q}, Q_{\text {int }}} \\
\mathrm{NACP}\end{array}$ & $\begin{array}{c}D_{w, M a r k u s} \\
\text { (usando } \\
16 \mathrm{MeV} \\
\text { como } Q_{\text {cross }} \text { ) }\end{array}$ & $\begin{array}{c}D_{w, N A C P} \\
\text { (usando } \\
16 \mathrm{MeV} \\
\text { como } Q_{\text {cross }} \text { ) }\end{array}$ & $\begin{array}{c}D_{w, N A C P} \\
\text { (usando } \\
20 \mathrm{MeV} \\
\text { como } Q_{\text {cross }} \text { ) }\end{array}$ \\
\hline 4 & 1,32 & 0,69 & $100 \%$ & 1,044 & 1,070 & 0,983 & 1,004 & 1,013 \\
\hline 6 & 2,39 & 1,33 & $100 \%$ & 1,034 & 1,049 & 0,998 & 1,005 & 1,014 \\
\hline 9 & 3,58 & 2,05 & $100 \%$ & 1,024 & 1,033 & 1,017 & 1,018 & 1,027 \\
\hline 12 & 5,06 & 2,94 & $100 \%$ & 1,014 & 1,018 & 1,007 & 0,999 & 1,018 \\
\hline 16 & 6,71 & 3,93 & $98,3 \%$ & 1,005 & 1,005 & 1,044 & 1,026 & 1,035 \\
\hline
\end{tabular}
e NACP-02.

Tabela 2 Características dos feixes de elétrons do acelerador Clinac 23EX e calibração dos feixes de energias

\begin{tabular}{|c|c|c|c|c|c|c|c|c|c|}
\hline $\begin{array}{l}\text { Energia } \\
(\mathrm{MeV})\end{array}$ & $\begin{array}{c}R_{50} \\
\text { (g. } \mathrm{cm}^{-2} \text { ) }\end{array}$ & $\begin{array}{c}z_{\text {ref }} \\
\left(\text { g. } \mathrm{cm}^{-2} \text { ) }\right.\end{array}$ & $\begin{array}{c}\text { PDP } \\
\text { em } z_{\text {ref }}\end{array}$ & $\begin{array}{c}k_{Q, Q_{\text {int }}} \\
\text { Markus }\end{array}$ & $\begin{array}{l}k_{Q, Q_{\text {int }}} \\
\text { NACP }\end{array}$ & $\begin{array}{c}D_{w, \text { Markus }} \\
\text { (usando } \\
16 \mathrm{MeV} \\
\text { como } Q_{\text {cross }} \text { ) }\end{array}$ & $\begin{array}{c}D_{w, N A C P} \\
\text { (usando } \\
16 \mathrm{MeV} \\
\text { como } Q_{\text {cross }} \text { ) }\end{array}$ & $\begin{array}{c}D_{w, N A C P} \\
\text { (usando } \\
20 \mathrm{MeV} \\
\text { como } Q_{\text {cross }} \text { ) }\end{array}$ & $D_{w, N E 2571}$ \\
\hline 4 & 1,18 & 0,61 & $100 \%$ & 1,045 & 1,074 & 1,006 & 1,019 & 1,028 & - \\
\hline 6 & 2,31 & 1,28 & $100 \%$ & 1,034 & 1,050 & 1,027 & 0,985 & 0,994 & - \\
\hline 9 & 3,53 & 2,02 & $100 \%$ & 1,024 & 1,034 & 1,002 & 1,003 & 1,012 & 0,999 \\
\hline 12 & 4,91 & 2,85 & $100 \%$ & 1,015 & 1,020 & 1,015 & 1,000 & 1,009 & 1,000 \\
\hline 16 & 6,55 & 3,83 & $99,1 \%$ & 1,005 & 1,006 & 1,054 & 1,004 & 1,013 & 1,005 \\
\hline 20 & 8,18 & 4,81 & $95,9 \%$ & 0,998 & 0,996 & 1,062 & 1,020 & 1,030 & 1,020 \\
\hline
\end{tabular}
de 16 e 20 MeV, respectivamente, usando as câmaras de placas paralelas Markus e NACP-02. 
cia para as energias de $9,12,16$ e $20 \mathrm{MeV}$. Observa-se que os resultados obtidos foram os mesmos que os encontrados com a câmara NACP-02 calibrada em um feixe de $16 \mathrm{MeV}$. Adicionalmente, quando o feixe de elétrons é calibrado na qualidade de referência (por exemplo, $16 \mathrm{MeV}$ ), o valor de $k_{Q, Q_{\text {int }}}$ terá um valor unitário. O mesmo raciocínio se aplica para a energia de $20 \mathrm{MeV}$.

Como teste final, foi determinada a dose absorvida na água empregando uma câmara cilíndrica tipo Farmer, modelo NE 2571, número de série 2194, para as energias de 9, 12, 16 e $20 \mathrm{MeV}$, respectivamente. Os resultados obtidos são apresentados na última coluna da Tabela 2. Observa-se que o resultado da calibração do feixe de elétrons com a câmara Farmer é o mesmo se for empregada a câmara de placas paralelas NACP calibrada em uma qualidade de referência de $16 \mathrm{MeV}$. Além disto, verifica-se a mesma diferença, entre $0,8 \%$ e $0,9 \%$, entre os valores de dose absorvida na água determinados com a câmara NACP, empregando uma $Q_{\text {cross }}$ de 16 e 20 $\mathrm{MeV}$, respectivamente.

\section{CONCLUSÕES}

As diferenças encontradas com a câmara Markus estavam de certa forma previstas, de acordo com os relatos encontrados na literatura. Variações de até $6 \%$ foram obtidas com tal câmara, demonstrando claramente um problema de construção. Este problema aparentemente foi solucionado com o lançamento de um novo modelo de câmara de placas paralelas, a Advanced Markus, a qual provavelmente teve um aperfeiçoamento no seu sistema de anel de guarda, fator responsável pelo alto fator de perturbação indicado pelo protocolo da IAEA $^{(9)}$. Recomenda-se aos hospitais que dispuserem desta câmara que façam uma análise comparativa, a exemplo do que foi feito no presente trabalho, a fim de verificar as possíveis discrepâncias, sobretudo durante a transição do protocolo anterior da IAEA $^{(6)}$ para o protocolo atual ${ }^{(7)}$.

Os valores de dose absorvida na água assim determinados com a câmara NACP no Clinac $2100 \mathrm{C}$ para uma $Q_{\text {int }}$ de $16 \mathrm{MeV}$ apresentam variação de $0,04 \%$ para a menor energia (4 MeV) e de $2,6 \%$ para a maior energia (16 MeV), em relação ao protocolo TRS-381. Já para uma $Q_{\text {int }}$ de 20 $\mathrm{MeV}$, as variações observadas são de $1,3 \%$ e $3,5 \%$. Por outro lado, as doses absorvidas na água determinadas no Clinac 23EX para as $Q_{\text {int }}$ de $16 \mathrm{MeV}$ e de $20 \mathrm{MeV}$ são de $1,9 \%$ e $2,0 \%$ e de $2,8 \%$ e $3,0 \%$, respectivamente. Conseqüentemente, a escolha de $Q_{\text {int }}$ não é tão crítica assim, principalmente quando não de dispõem de feixes de elétrons que tenham na prática um valor de $R_{50}$ exatamente igual a 7,5 g.cm ${ }^{-2}$.

\section{REFERÊNCIAS}

1. International Commission on Radiation Units. Determination of absorbed dose in a patient irradiated by beams of X or gamma rays in radiotherapy procedures. Bethesda: ICRU, 1976;24.
2. Reich H. Choice of the measuring quantity for therapy-level dosemeters. Phys Med Biol 1979;24: 895-900.

3. Hohlfeld K. The standard DIN 6800. Procedures for absorbed dose determination in radiology by the ionization method. In: Dosimetry in Radiotherapy 1988;13-24. Vol I, IAEA-SM-298/31. Vienna: IAEA.

4. Deutsches Institut für Normung. Dosismessverfahren in der radiologischen technik ionisationsdosimetrie. Norm DIN 6800, 1991; Teil 2.

5. (No authors listed). Procedures in external radiation therapy dosimetry with electron and photon beams with maximum energies between 1 and $50 \mathrm{MeV}$. Recommendations by the Nordic Association of Clinical Physics (NACP). Acta Radiol Oncol 1980;19:55-79.

6. International Atomic Energy Agency. Absorbed dose determination in photon and electron beams. 2nd ed. Technical Report Series No. 277. Vienna: IAEA, 1997.

7. International Atomic Energy Agency. Absorbed dose determination in external beam radiotherapy. Technical Report Series No. 398. Vienna: IAEA, 2000.

8. International Atomic Energy Agency. Absorbed dose determination in photon and electron beams. Technical Report Series No. 277. Vienna: IAEA, 1987.

9. International Atomic Energy Agency. The use of plane parallel ionization chambers in high-energy electron and photon beams. An International Code of Practice for Dosimetry. Technical Report Series No. 381. Vienna: IAEA, 1997.

10. Mattsson LO, Johansson KA, Svensson H. Calibration and use of plane-parallel ionization chambers for the determination of absorbed dose in electron beams. Acta Radiol Oncol 1981;20:385-399.

11. International Atomic Energy Agency. Errata list and updates to TRS-398 (2000), Vienna: IAEA.

12. Andreo P, Huq MS, Westermark M, et al. Protocols for the dosimetry of high-energy photon and electron beams: a comparison of the IAEA TRS-398 and previous international codes of practice. International Atomic Energy Agency. Phys Med Biol 2002;47:3033-3053. 